\title{
Advanced Materials for High Performance Fuel Cells and Electrolyzers
}

\author{
Aimy Bazylak ${ }^{1^{*}}$ \\ ${ }^{1}$ Thermofluids for Energy and Advanced Materials (TEAM) Laboratory, Department of Mechanical and Industrial \\ Engineering, University of Toronto
}

\begin{abstract}
Polymer electrolyte membrane (PEM) fuel cells and electrolyzers are composed of porous materials, including the catalyst layer, microporous layer, and substrate. Commercial materials, whether by design or not, typically exhibit highly heterogeneous material and chemical properties. In order to reach cost targets for widespread commercial adoption, we must realize materials that enable more effective multiphase flow phenomena than what currently exists. Mass transport losses in PEM fuel cells and electrolyzers are both prohibitively significant, yet designing these materials requires the a priori knowledge of how the heterogeneous properties of the porous materials and their interfacial contacts influence electrochemical performance. I will discuss these critical design factors (heterogeneous porous materials and nature of interfacial contacts) and how they influence the flow and mass transport behaviour in PEM fuel cells and electrolyzers. I will also discuss the new materials we have designed and fabricated, informed by in-house numerical modelling and tested through a combination of in operando and ex situ X-ray and neutron beam characterization approaches.
\end{abstract}

